\title{
Correspondence
}

\section{Psychiatrists in training}

Sir: Regarding the complaint of P. De Vries (Psychiatric Bulletin, 20, 52-53) that psychiatrists in training are often tormented by the need to pass professional examinations and at the same time develop patient care skills, I cannot see a conflict between preparing for the MRCPsych, and improving all aspects of clinical practice. The knowledge base acquired has clear value in day to day patient care and encourages an informed and reasoned approach.

The pressure to pass higher examinations ensures we become familiar with this important body of knowledge. The MRCPsych examination includes an evaluation of the candidate's patient care skills. I have found these skills most noticeable by their absence in trainees who go on to fail. Passing the membership is the beginning of stressful competition for posts and guidance can seem inadequate at times.

I would reject as cynical and sensational the statement that many who pass the membership become self-seeking or burnt out. Doctors in all branches of medicine are subject to considerable stress and job satisfaction appears to be at an all time low. There are other more obvious causes for this than professional examinations such as low pay, changes to working practices, underfunding of community care, and balancing responsibilities to our families and patients.

The examination for MRCPsych is under constant review and is not in need of replacement with inferior tests of trainees' abilities.

B. C. Timmins, Duchess of Kent Hospital, North Yorkshire DL9 4DF

Sir: We have to agree with quite a few of $\mathrm{Dr}$ Timmins' comments. The knowledge required to pass the MCQ examination 'may' have value in patient care but does not 'commonly'. For instance " $\mathrm{A}$ patient being treated with a MAOI should not eat A: broad bean pods; B: banana skins ..." No patient has ever asked us an MCQ!

Regarding patient care skills, we are not entirely clear what makes a 90 minute clinical examination 'superior' to 6 months' continuous evaluation with a supervising consultant? We are making no attempts to be "cynical and sensational" but sadly there is a stronger correlation between pass rate and exam oriented training than with patient care skill or experience.

P. DE VRIES, Fulbourn Hospital, Cambridge CB1 5EF; A. MICHAEL, Department of Psychiatry, Addenbrooke's Hospital, Cambridge CB2 $29 Q$

\section{"Toys for the boys"}

Sir: The Registrar of the College has written about the Fellowship (Psychiatric Bulletin, 20, 185187). One possibility left unconsidered is the abolition of the Fellowship or "Toys for the Boys" as it might well be called.

Is it really necessary to have this distinction? It is clear that the Fellowship is a self-perpetuating oligarchy which will tend to exclude those who have a low profile on the national or regional scene, but may still be doing good work in their locality.

Fortunately, the only privilege of the Fellowship is to pay an increased subscription to the College and to have the opportunity to stand for President. Neither of these will have much appeal to many members! Although it could be argued that excluding members of the College from election to high office is fundamentally undemocratic and ensures that senior officers of the College can never be truly representative of the membership.

I propose the abolition of the grades of member and Fellow and would welcome suggestions for an appropriate new all-encompassing title which would be won by examination.

\section{A. Molver, Charlton Lane Centre, Cheltenham, Gloucestershire GL53 9DZ}

\section{The use of high-dose antipsychotics in rapid tranquillisation}

Sir: Hillam \& Evans have reported that the majority of patients in an ICU were receiving antipsychotic drugs in doses exceeding the BNF recommended maximum doses and that polypharmacy was the rule rather than the exception (Psychiatric Bulletion, 20, 82-84).

In their survey of rapid tranquillisation, Simpson \& Anderson (Psychiatric Bulletin, 20. 149-152) state that most psychiatrists use sensible drug regimes for rapid tranquillisation. However, the median maximum doses which their respondents would give were close to the BNF maxima, implying that half would consider exceeding these doses. At the same time they note that many psychiatrists believe that 